\title{
Detection of the artery of Adamkiewicz using multidetector row computed tomography in patients with spinal arteriovenous shunt disease
}

\author{
Sodai Hoshiai ${ }^{1 A, B, C, D, E, F}$, Masanari Shiigai ${ }^{2 A, B, D, F}$, Takahiro Konishi2A,B,F, Yasunobu Nakai ${ }^{3 A, B, E}$, Tomohiko Masumoto ${ }^{1 A, B, C, E, F}$ \\ 'Department of Radiology, Faculty of Medicine, University of Tsukuba, Tsukuba, Ibaraki, Japan \\ 2Department of Radiology, Tsukuba Medical Centre Hospital, Tsukuba, Ibaraki, Japan \\ ${ }^{3}$ Intravascular Treatment Centre, Yokohama Brain and Spine Centre, Isogo-ku, Yokohama, Japan
}

\section{Abstract}

Purpose: To plan a treatment strategy for a spinal arteriovenous shunt (SAVS), identifying the artery of Adamkiewicz (AKA) and its origin is indispensable. However, detecting the AKA is very difficult in patients with an SAVS when using computed tomography angiography (CTA) by the usual method to find the hairpin curved artery because dilated drainage veins nearly always coexist with the hairpin curved AKA. We designed a method to identify the AKA by focusing on the diameter and pathway of the anterior radiculomedullary arteries (RMAs).

Material and methods: Seven consecutive patients with an SAVS were surveyed. They underwent contrast-enhanced CTA and conventional angiography from January 2009 to December 2012. Two readers evaluated the CTA images and assumed that the AKA was the artery that ran through the anterior portion of the neural foramen and continued to pass on the ventral side of the spinal cord.

Results: Among the seven patients, nine AKAs were detected with conventional angiography. When using our method, seven AKAs and six AKAs were identified on CTA by Reader 1 and Reader 2, respectively. The average sensitivity was $72.3 \%$, and the specificity, accuracy, positive predictive value, and negative predictive value were sufficiently high (i.e. > 85\%) for both readers. The kappa value for detecting the AKA was 0.98 .

Conclusions: Detecting the origin of the AKA with CTA is challenging in patients with an SAVS. However, focusing on the diameter and pathway of the RMAs may allow successful identification.

Key words: artery of Adamkiewicz, multidetector computed tomography, computed tomography, computed tomography angiography, spinal arteriovenous shunt, arteriovenous fistula.

\section{Introduction}

The artery of Adamkiewicz (AKA), also called the great anterior radiculomedullary artery (RMA), is the dominant RMA arising from the thoracolumbar segmental artery that supplies the anterior spinal artery [1]. Accurate localisation of the AKA in patients with a spinal arteriovenous shunt (SAVS) is extremely important before planning their treatment strategy $[2,3]$. If a feeding artery of an SAVS originates at the same level as the AKA, endovascular treatment is a significant contraindication because of the high risk of severe neurological complications [2]. Occlusion of the AKA, which continues to the anterior spinal artery, is dangerous and must be avoided under all conditions. Predicting the location of the AKA may also prevent complications during selective intercostal or lumbar angiography [4].

Owing to advances in imaging techniques, noninvasive assessment of the AKA by using computed tomography angiography (CTA) or magnetic resonance angiography has been developed [5-7]. CTA or magnetic resonance angiography can successfully depict the characteristic hairpin curve on the midline ventral surface of the spinal cord of the AKA. Finding the characteristic hairpin curve is important for detecting the AKA $[5,6,8,9]$; how-

Correspondence address:

Dr. Sodai Hoshiai, Department of Radiology, Faculty of Medicine, University of Tsukuba, 1-1-1 Tennodai, Tsukuba, Ibaraki 305-8575, Japan,

e-mail: hoshiai@md.tsukuba.ac.jp

Authors' contribution:

A Study design · B Data collection · C Statistical analysis · D Data interpretation · E Manuscript preparation · F Literature search · G Funds collection 


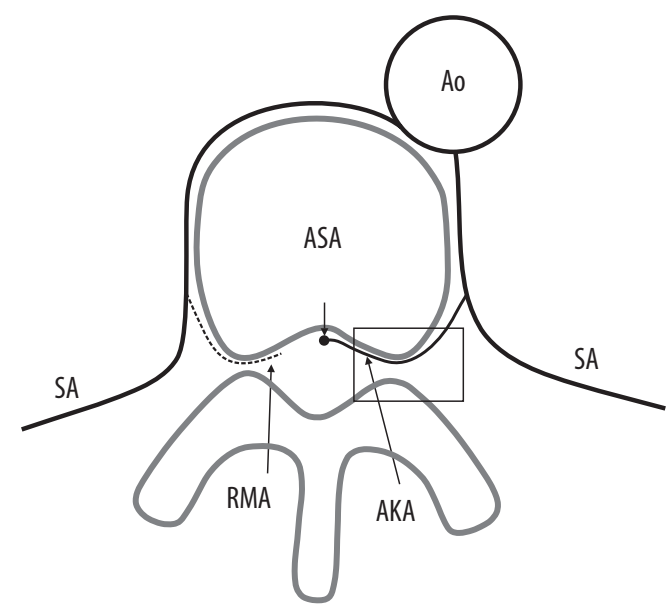

A0 - aorta, ASA - anterior spinal artery, SA - segmental artery, RMA - radiculomedullary artery (other than the AKA), AKA - artery of Adamkiewicz

Figure 1. The diagram shows the radiculomedullary artery (RMA) arising from the segmental artery. The artery of Adamkiewicz (AKA) is the dominant RMA, arising from the thoracolumbar segmental artery that supplies the anterior spinal artery. The AKA has a larger diameter than the other RMAs and runs along the anterior nerve root. In the region of the rectangle, the AKA is the largest RMA that runs through the ventral aspect of the neural foramen and continues to pass on the ventral side of the spinal cord

ever, the AKA is very difficult to detect in patients with an SAVS because abundant dilated drainage veins nearly always coexist with the hairpin curved AKA.

The RMAs course along the ventral aspect of individual spinal nerve roots, pierce the dura, and distribute to the anterior nerve roots [10]. The diameter of the AKA is larger than that of other RMAs [11]. The largest artery that runs the ventral aspect of the neural foramen is presumed to be the AKA. In this study, we assessed the ability to detect the AKA in patients with an SAVS by using CTA to focus on the diameter and pathway of RMAs originating from thoracic or lumbar segmental arteries.

\section{Material and methods}

\section{Patients}

This retrospective study was approved by our institutional review board, which waived the need for informed consent. Seven consecutive patients (five men and two women; age range, 55-77 years; mean age, 64.9 years), who underwent CTA and conventional selective spinal angiography for an SAVS between January 2009 and December 2012, were enrolled in the study. The SAVSs included four spinal dural arteriovenous fistulas, two filum terminale arteriovenous fistulas, and one spinal epidural arteriovenous fistula.

\section{Computed tomography angiography data acquisition and spinal angiography}

All patients were imaged in the supine position by using a 64-detector CT system (Brilliance 64; Philips Medical Systems, Amsterdam, Netherlands) from the apex of the lungs to the pubic symphysis. In the bolus-tracking method, the region of interest (ROI) was in the descending aorta at the level of the celiac artery. Image acquisition was started 10 seconds after the CT value of the ROI reached its threshold, which was set at 150 Hounsfield units. $100 \mathrm{ml}$ iopamidol (Iopamiron 370, 370 mg I/ml; Bayer, Leverkusen, Germany) was administered at a rate of $4 \mathrm{ml} / \mathrm{s}$ through a 20-gauge intravenous cannula, which was placed in the right antecubital vein, followed by a $25 \mathrm{ml}$ physiologic saline flush at the same rate using a dual-head power injector (Dual Shot GX; Nemoto Kyorindo, Tokyo, Japan). Reconstruction was conducted in the axial plane of the CTA image data with $1.0 \mathrm{~mm}$ thickness and $0.5 \mathrm{~mm}$ interval.

Selective intercostal and lumbar artery catheterisation was conducted using a 4-Fr hook-shaped or cobra catheter via right femoral artery access. The contrast medium was manually injected. Digital subtraction angiography (DSA) images were obtained with angiographic equipment (Integris v5000 or Allura Xper FD20/20; Philips Medical Systems, Amsterdam, Netherlands).

\section{Objective image analysis}

Two board-certified radiologists (S.H. with 12 years of experience and M.S. with 18 years of experience), who were blind to the results of conventional selective angiography, evaluated all CTA images by using commercial viewing software (Centricity RA1000; GE Healthcare, Barrington, IL, USA). They could adjust the window level and width and browse the multiplanar reconstruction images or view maximum intensity projection (MIP) images. They considered the largest RMA as the AKA, which ran through the ventral aspect of the neural foramen and continued to pass on the ventral side of the spinal cord (Figure 1). The radicular vein was distinguished by the absence of continuity from the aorta or by a difference in contrast enhancement. The feeding artery of the SAVS was distinguished by continuity of the shunt point or venous pouch.

\section{Statistical analysis}

The diagnostic performance of the two readers was assessed by calculating sensitivity, specificity, accuracy, positive predictive value (PPV), and negative predictive value (NPV) in the total number of segmental arteries identified by conventional selective angiography. Interobserver agreement was assessed by using kappa statistics.

\section{Results}

Conventional selective angiography depicted 122 segmental arteries, which are listed in Table 1. A single AKA was identified in five patients with DSA, and double AKAs were identified in two patients. Conventional angiography revealed nine AKAs in total. The AKA originated from the left segmental artery in eight of nine AKAs and the re- 
Table 1. The identification of the artery of Adamkiewicz with conventional selective angiography and the results of two readers in identifying the artery of Adamkiewicz by using computed tomography angiography

\begin{tabular}{|c|c|c|c|c|c|c|c|c|}
\hline \multirow[t]{2}{*}{ Patient } & \multirow[t]{2}{*}{ Age $(y)$} & \multirow[t]{2}{*}{ Sex } & \multirow[t]{2}{*}{$\begin{array}{l}\text { Level of the } \\
\text { AKA origin }\end{array}$} & \multicolumn{2}{|c|}{$\begin{array}{l}\text { Segmental arteries evaluated } \\
\text { with selective angiography } \\
\text { ( } n=122)\end{array}$} & \multirow[t]{2}{*}{ Disease } & \multirow[t]{2}{*}{ Reader 1} & \multirow[t]{2}{*}{ Reader 2} \\
\hline & & & & Right & Left & & & \\
\hline 1 & 77 & Male & Right L1 & T8-L3 (8) & T8-L3 (8) & edAVF & Detected & ND \\
\hline 2 & 55 & Male & Left T7 & T4-L1, L3-4 (12) & T5-L3 (11) & $\mathrm{ft}$ AVF & Detected & Detected \\
\hline 3 & 68 & Female & Left L2 & T7-L4 (10) & T7-11, L1-4 (9) & dAVF & ND & ND \\
\hline 4 & 67 & Female & Left L1 & T4-L4 (13) & T4-L4 (13) & $\mathrm{ft}$ AVF & Detected & Detected \\
\hline 5 & 64 & Male & $\begin{array}{l}\text { Left T9 } \\
\text { Left L2 }\end{array}$ & T8-11, L1-3 (7) & T6-L2 (9) & dAVF & $\begin{array}{l}\text { Detected } \\
\text { ND }\end{array}$ & $\begin{array}{c}\text { Detected } \\
\text { ND }\end{array}$ \\
\hline 6 & 59 & Male & Left T9 & T6-9 (4) & T6-9 (4) & dAVF & Detected & Detected \\
\hline 7 & 64 & Male & $\begin{array}{c}\text { Left T8 } \\
\text { Left T10 }\end{array}$ & T4, T6-11 (7) & T6-12 (7) & dAVF & $\begin{array}{l}\text { Detected } \\
\text { Detected }\end{array}$ & $\begin{array}{l}\text { Detected } \\
\text { Detected }\end{array}$ \\
\hline
\end{tabular}

AKA - artery of Adamkiewicz, edAVF - epidural arteriovenous fistula, ND - not detected, L - lumbar segmental artery, ft AVF - filum terminale arteriovenous fistula, $\mathrm{T}$ - thoracic segmental artery, dAVF - dural arteriovenous fistula

The number of angiographically enhanced arteries is in parentheses.

maining AKA originated from the right segmental artery, which ranged from the level of the seventh intercostal to the second lumbar artery. Two AKAs contributed partly as feeding arteries in two patients with filum terminale arteriovenous fistulas, whereas the other AKAs did not contribute as feeding arteries.

Reader 1 correctly detected seven of nine AKAs by using CTA and did not indicate any false-positive arteries. Reader 2 correctly identified six of nine AKAs and falsely recognised one feeding artery of SAVS as the AKA with CTA. Both readers identified double AKAs in one patient with CTA. In another patient with a double AKA, each reader could identify only one AKA with CTA. The characteristic hairpin curve on the midline ventral surface of the spinal cord was not depicted in all patients because it may have blended into the abundant dilated shunt vessels. The sensitivity, specificity, accuracy, PPV, and NPV of detecting the AKA are summarised in Table 2. The sensitivities were $77.8 \%$ and $66.7 \%$ for readers 1 and 2 , respectively. The specificity, accuracy, PPV, and NPV were sufficiently high (i.e. $>85 \%$ ) for both readers. The total accuracy of the two readers was $97.6 \%$. The kappa value for detecting the AKA was 0.98, which indicated nearly perfect interobserver agreement.

\section{Representative case}

Figures 2 and 3 show representative CTA images. The oblique coronal MIP image along the thoracolumbar spine of the patient with the dural arteriovenous fistula shows dilated drainage veins (Figure 2A). When using the method of finding the characteristic hairpin curve, accurate localisation of the AKA was extremely difficult because of surrounding dilated drainage veins. The curved coronal planar reformation image along the left eighth
Table 2. Diagnostic value for detecting the artery of Adamkiewicz by using computed tomography angiography

\begin{tabular}{|l|c|c|} 
& Reader 1 & Reader 2 \\
\hline Sensitivity & $77.8 \%$ & $66.7 \%$ \\
\hline Specificity & $100 \%$ & $99.1 \%$ \\
\hline Accuracy & $98.4 \%$ & $96.7 \%$ \\
\hline PPV & $100 \%$ & $85.7 \%$ \\
\hline NPV & $98.3 \%$ & $97.4 \%$ \\
\hline
\end{tabular}

PPV - positive predictive value, NPV - negative predictive value

and tenth intercostal artery delineates two RMAs entering the vertebral canal (Figure 2B). Axial sliding MIP images at the level of the left eighth intercostal artery allows visualisation of the continuity of the left eighth intercostal artery and RMA, which runs along the ventral aspect of the neural foramen (Figure 3A). Axial MIP images at the level of the left $10^{\text {th }}$ intercostal artery shows a similar finding (Figure 3B). The two readers considered these two arteries as the AKA. Axial sliding MIP images at the level of right sixth intercostal artery shows a feeding artery, which continues a dilated drainage vein via a shunt point (Figure 3C). All sequential axial images are available as a video in the Electronic Supplementary Material: https:// vimeo.com/398179381/1a55e46583. No other arteries running into the vertebral canal were detected, except for the three aforementioned arteries.

Figure 4 shows representative DSA images of the same patient in Figures 2 and 3. Conventional selective angiography of the left eighth and tenth intercostal arteries depicts RMAs continuing into the anterior spinal arteries in Figure 4A and Figure 4B, respectively. Both figures depict the characteristic hairpin curve, which indicates that these RMAs are AKAs. 


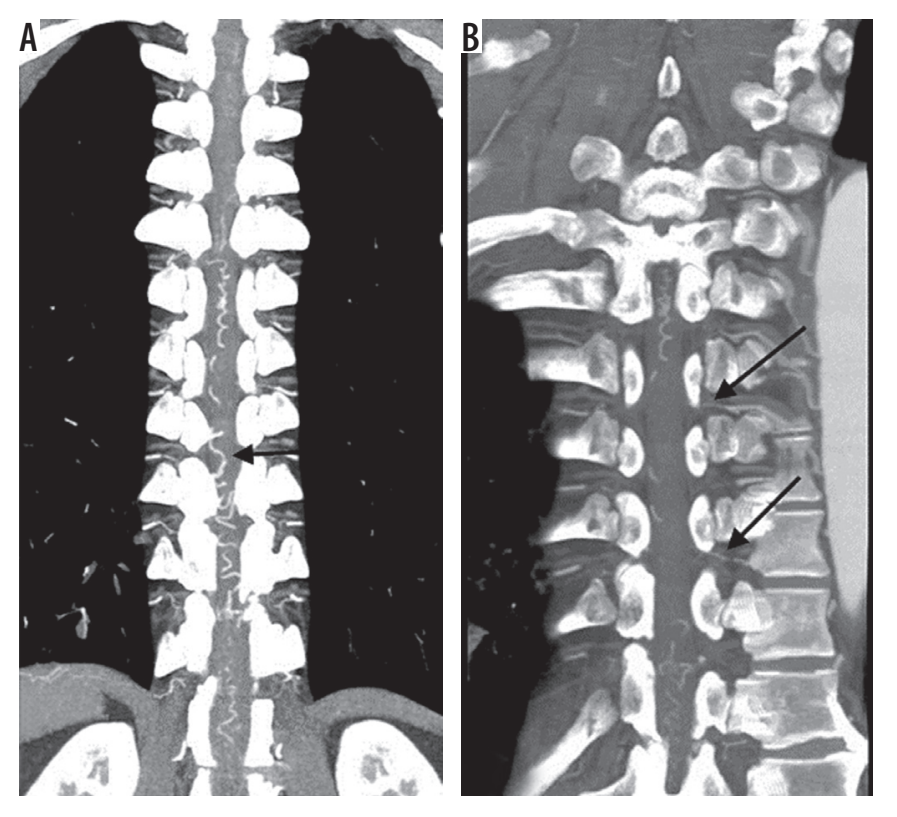

Figure 2. A) The oblique coronal maximum intensity projection (MIP) image of computed tomography angiography (CTA) along the thoracolumbar spine of a patient with a dural arteriovenous fistula. The characteristic hairpin curve of the Adamkiewicz artery on the midline ventral surface of the spinal cord is not depicted because of the surrounding dilated drainage veins (arrow). B) The curved coronal planar reformation image along the left eighth and tenth intercostal artery delineates two radiculomedullary arteries (arrows) entering the vertebral canal

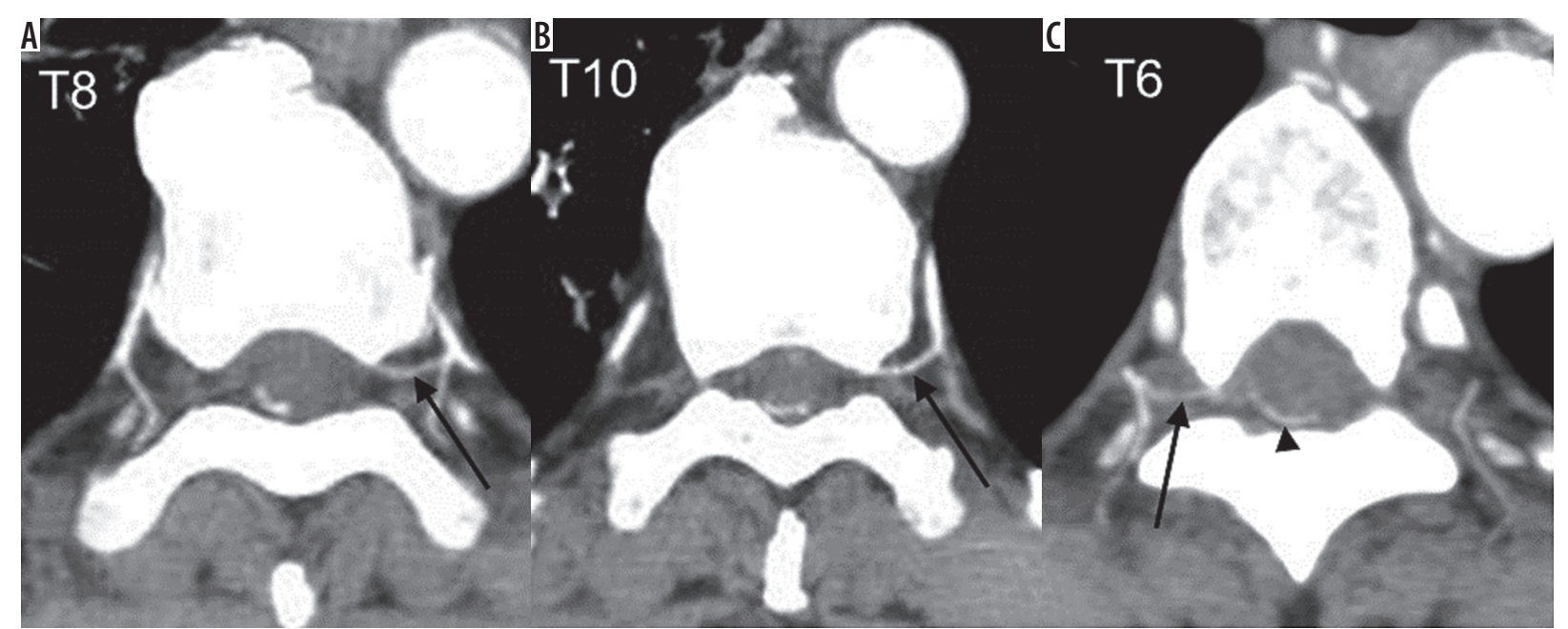

Figure 3. A) The axial maximum intensity projection (MIP) image at the level of left eighth intercostal artery allows the visualisation of the continuity of the left eighth intercostal and radiculomedullary artery (arrow), which runs along the ventral aspect of the vertebral canal. B) The axial MIP image at the level of the left tenth intercostal artery (arrow) also shows a similar finding. C) The axial MIP image at the level of the right sixth intercostal artery shows a feeding artery (arrow), which is continuing a dilated drainage vein (arrowhead) via a shunt point

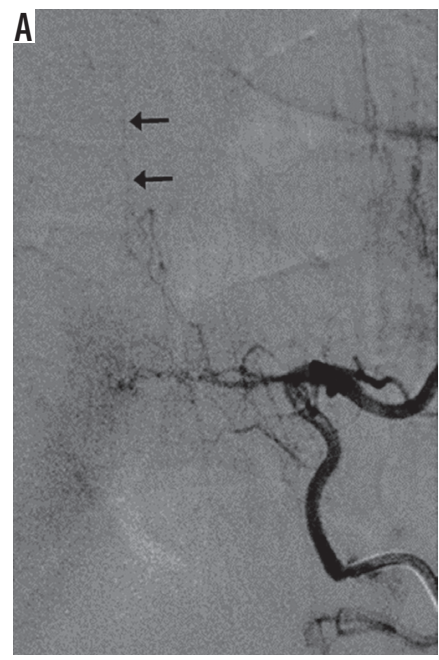

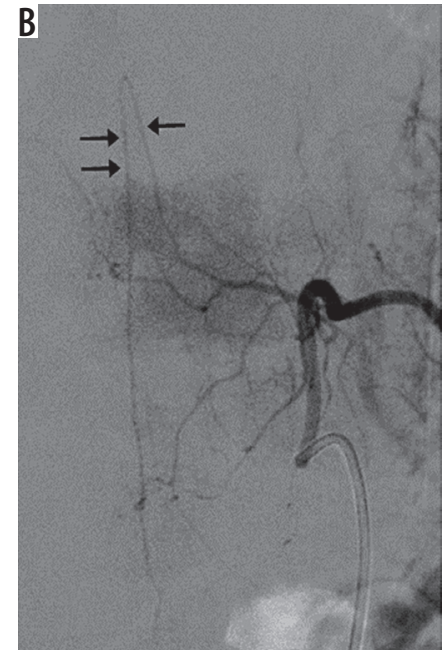

Figure 4. Conventional selective angiography of the left (A) eighth and (B) tenth intercostal arteries depicts radiculomedullary arteries continuing the anterior spinal arteries on the midline of the spine. Both figures show the characteristic hairpin curve and continuation to the anterior spinal artery, which indicates that these radiculomedullary arteries are arteries of Adamkiewicz 


\section{Discussion}

The AKA is the most important feeding artery of the thoracolumbar spinal cord. This artery supplies the lower one-third of the spinal cord. The AKA, which is the largest RMA, courses along the nerve root to reach the anterior surface of the spinal cord and provides a major arterial supply to the spinal cord [10]. The ascending course of the AKA and descending ramus creates the characteristic appearance of a hairpin curve.

Several past studies $[5,6,8]$ have reported that detecting a specific hairpin-curved artery on the ventral surface of the spinal cord is important to localise the AKA with CTA. However, this detection is difficult in a patient with SAVS because of the presence of abundant dilated vessels due to arteriovenous shunts. However, accurate localisation of the AKA in a patient with SAVS is very important to plan a treatment strategy. In this study, we assessed AKA localisation using CTA in patients with an SAVS by focusing on the diameter and pathway of arteries originating from the segmental artery and running through the anterior aspect of the neural foramen. The sensitivity of detecting the AKA with CTA by the two readers was $77.8 \%$ and $66.7 \%$. The total accuracy of the two readers was sufficiently high (97.6\%) when using our method.

Several studies have reported the depiction of the AKA with CTA and reported a rate of visualisation of 68$90 \%[5,6,13]$. Their results seem to be superior to those of our study. However, accurate localisation of the AKA may not have been established because previous studies [8,13-15] did not conduct spinal conventional selective angiography. The most reliable way to visualise the AKA is with conventional selective arterial angiography [16]. In this study, localisation of the AKA was established with selective angiography. Conventional selective spinal angiography also revealed that two AKAs existed in two (29\%) of seven patients. This finding is consistent with the results of an autopsy study [17] in which two AKAs were identified in $26 \%$ of cadavers examined. Two AKAs were identified by using CTA with our method in one of two patients.
Our method must be useful to plan the strategy of treating SAVSs safely, especially in endovascular treatment. Even conventional selective spinal angiography that includes no treatment may cause critical complications such as paraplegia during the procedure [4]. Localisation of the AKA beforehand may reduce the risk of ischaemic spinal complications during conventional angiography, owing to the careful handling of catheters and gentle injection of the contrast material required for angiography of the AKA.

We acknowledge that our study has some limitations because of its single-institution retrospective design. First, all patients in our study were Asian and had a relatively small body size. The contrast material volume and scan method may not be applied to obese patients. Second, the characteristics of our study restricted the number of objective patients. Seven patients with an SAVS underwent both CTA and conventional selective angiography. Further validation is needed to improve the precision of our study, although our study adequately revealed a high accuracy in detecting AKA. Third, the two AKAs that partly contributed as feeding arteries in patients with filum terminale arteriovenous fistula might have been dilated and thus easily identifiable, although the diameters of those feeder AKAs were not lager than the other AKAs on conventional angiography images. Finally, the specificity and the NPV may have been estimated as high, owing to the characteristics of statistics, because the frequency of the AKA in the segmental arteries was very low.

\section{Conclusions}

Focusing on the continuity of an artery from the segmental artery and its course through the anterior portion of the neural foramen is useful for detecting the AKA, even in patients with an SAVS. This method may be useful when preparing a patient for selective arterial angiography and for planning the treatment of SAVS.

\section{Conflict of interest}

The authors report no conflict of interest.

\section{References}

1. Yoshioka K, Niinuma H, Ehara S, et al. MR angiography and CT angiography of the artery of Adamkiewicz: state of the art. Radiographics 2006; 26 (Suppl 1): S63-S73.

2. Rodesch G, Lasjaunias P. Spinal cord arteriovenous shunts: from imaging to management. Eur J Radiol 2003; 46: 221-232.

3. Geibprasert S, Pereira V, Krings T, et al. Dural arteriovenous shunts: a new classification of craniospinal epidural venous anatomical bases and clinical correlations. Stroke 2008; 39: 2783-2794.

4. Kieffer E, Fukui S, Chiras J, et al. Spinal cord arteriography: a safe adjunct before descending thoracic or thoracoabdominal aortic aneurysmectomy. J Vasc Surg 2002; 35: 262-268.
5. Takase K, Sawamura Y, Igarashi K, et al. Demonstration of the artery of Adamkiewicz at multi- detector row helical CT. Radiology 2002; 223: 39-45.

6. Kudo K, Terae S, Asano T, Oka M, et al. Anterior spinal artery and artery of Adamkiewicz detected by using multi-detector row CT. Am J Neuroradiol 2003; 24: 13-17.

7. Yamada N, Takamiya M, Kuribayashi S, et al. MRA of the Adamkiewicz artery: a preoperative study for thoracic aortic aneurysm. J Comput Assist Tomogr 2000; 24: 362-368.

8. Yoshioka K, Tanaka R, Takagi H, et al. Ultra-high-resolution CT angiography of the artery of Adamkiewicz: a feasibility study. Neuroradiology 2018; 60: 109-115. 
9. Kubota Y, Yokota H, Mukai H, et al. Low-tube-voltage CT assessment of Adamkiewicz artery: Precise comparison between $100-\mathrm{kVp}$ and 120-kVp protocols. Eur J Radiol 2019; 111: 56-61.

10. Takahashi S. Vessels of the spine and spinal cord: normal anatomy. In: Takahashi S (ed.). Neurovascular imaging. Springer, London 2010; 425-450.

11. Thron A. Anatomy of the spinal cord's blood supply. In: Thron A. Vascular anatomy of the spinal cord. Neuroradiological investigations and clinical syndromes. Wien New York, Springer 1988; 8-12.

12. Utsunomiya D, Yamashita Y, Okumura S, et al. Demonstration of the Adamkiewicz artery in patients with descending or thoracoabdominal aortic aneurysm: optimization of contrast-medium application for 64-detector-row CT angiography. Eur Radiol 2008; 18: 2684-2690.

13. Nakayama Y, Awai K, Yanaga Y, et al. Optimal contrast medium injection protocols for the depiction of the Adamkiewicz artery using 64-detector CT angiography. Clin Radiol 2008; 63: 880-887.
14. Shimoyama S, Nishii T, Watanabe $\mathrm{Y}$, et al. Advantages of $70-\mathrm{kV}$ CT angiography for the visualization of the Adamkiewicz artery: comparison with 120-kV imaging. Am J Neuroradiol 2017; 38 : 2399-2405

15. Nishii T, Kotoku A, Hori Y, et al. Filtered back projection revisited in low-kilovolt computed tomography angiography: sharp filter kernel enhances visualization of the artery of Adamkiewicz. Neuroradiology 2019; 61: 305-311.

16. Uotani K, Yamada N, Kono AK, et al. Preoperative visualization of the artery of Adamkiewicz by intra-arterial CT angiography. Am J Neuroradiol 2008; 29: 314-318.

17. Koshino T, Murakami G, Morishita K, et al. Does the Adamkiewicz artery originate from the larger segmental arteries? J Thorac Cardiovasc Surg 1999; 117: 898-905.

\section{Electronic Supplementary Material}

Electronic supplementary material 1: The video shows sequential axial images of computed tomography angiography 